\title{
Localized Asymmetry in Human Dental Crown Form- an Interesting Case
}

\author{
John Wetherell, Tracey Winning and Grant Townsend* \\ Dental School, The University of Adelaide, South Australia 5005
}

ABSTRACT. A case of a 20-year-old female is described in which the premolars and molars on the right side of the arch display altered crown proportions and altered occlusal morphology. There is no evidence of an orofacial congenital disorder or history of trauma. It is argued that the asymmetrical expression of crown form does not fall within the normal range of variation but has resulted from a localized disruption

This case highlights the fact that asymmetries in dental crown form, whether they be fluctuating or directional, need to be viewed as resulting from a continuum of developmental disturbances that may range from minor to severe. As our knowledge of the molecular basis of dental development continues to grow, we should eventually be able to explain in cellular and molecular terms the specific causes of the whole range of asymmetrical expressions in dental crown form that we observe within the human dentition.

The phenotypic appearance of newly-emerged dental crowns results from an interplay between an individual's genotype and environmental influences operating during the period of odontogenesis. Environmental factors may also alter crown appearance after teeth emerge into the oral cavity, for example due to trauma, caries or wear. However, careful examination of teeth intra-orally or indirectly via dental models will normally enable the examiner to distinguish between those crown variations that have occurred during development compared with those that have resulted after emergence.

It is generally assumed that the genetic influences operating on antimeric tooth pairs are identical so, in the absence of post-emergence effects, differences in crown morphology between corresponding teeth on opposite sides of the dental arch can be considered to reflect the influence of developmental disturbances during odontogenesis. These disturbances may vary in their timing, duration and severity.

Asymmetry in dental crown size is referred to as being directional if there is a tendency for dimensions on one side to be consistently larger than those of their corresponding antimeres. There is some evidence of

Editor's note: It is hoped that this case report will stimulate some productive discussion in the Journal. Please submit comments to the Editor. in cellular function within the developing tooth germs, probably upsetting the folding of the internal enamel epithelia. This has produced crowns that have rounded cuspal outlines and reduced intercuspal distances. Superimposed space constraints in the mandible may have also led to compression of the lower molar crowns mesiodistally and affected their root formation. Dental Anthropology 2004;17(1):18-23.

directionality in deciduous and permanent crown size in relatively large human samples that exclude individuals with major developmental disorders (Harris, 1992; Townsend et al., 1999). However, whether these findings reflect real underlying biological influences or represent chance effects remains unclear.

There also are various pathological conditions that may lead to directional asymmetries in dental crown size and shape. For example, in hemifacial microsomia-a developmental abnormality affecting the first and second branchial arches - the posterior teeth are smaller than normal, with the reduction in size being most marked on the affected side (Seow et al., 1998). This is an example of directional asymmetry where the affected teeth are smaller on the affected side.

Fluctuating dental asymmetry refers to the small random differences in crown size or morphology commonly observed between antimeric tooth pairs. These differences may be due, for example, to differences in blood supply or space availability between sides. More severe space constraints leading to distortion of developing tooth germs may result in compression of a tooth or teeth on one side producing more marked asymmetry in size and/or shape.

The magnitude of fluctuating dental asymmetry is increased in laboratory animals exposed to external stressors during development (Siegel et al., 1977) and in certain human chromosomal disorders, for example Down syndrome, where the aneuploidy is thought to disrupt homeostasis, leading to increased developmental instability (Townsend, 1983). A similar explanation has been put forward to account for increased fluctuating asymmetry in crown size noted in individuals with

*Address for correspondence: Grant Townsend, Dental School, The University of Adelaide, South Australia 5005 Email: grant.townsend@adelaide.edu.au 
sex chromosomal aneuploidies (Townsend et al., 1986) and in individuals with cleft lip and palate (Narayanan et al., 1999). Of considerable interest is the report of increased directional asymmetry in the occlusal morphology of permanent first molars in $45, \mathrm{X} / 46, \mathrm{X}$ mosaics (Pirttiniemi et al., 1998). This study indicates that different cell lines regulated by different genes may be responsible for differences in crown form on opposite sides of the dental arches.

In this paper we report on an interesting example of dental asymmetry that is evident in the maxillary and mandibular posterior segments of the permanent dentition of a young woman who has no history or signs of orofacial trauma or a congenital disorder. This case provides a good opportunity to ponder on how factors that have presumably operated unilaterally on the developing dental arches can lead to marked asymmetries in final crown forms in an otherwise healthy person.

\section{CASE REPORT}

Figures 1 and 2 show occlusal views of the maxillary and mandibular dental arches of a 20-year-old female of European ancestry who presented at the Adelaide Dental School in 2001 for a routine dental check-up. The woman had no history of any major medical problems, nor was there any history of her mother suffering illhealth during pregnancy. She had chicken pox as an 8-9 year-old but did not take any medication at that time. There was also no history or evidence of visible facial asymmetry.

In both arches, the first premolars had been extracted previously for orthodontic reasons, and the third molars had not emerged in the maxilla. The woman had also worn an upper removable orthodontic appliance for seven months in 1995. A supernumerary tooth had been extracted from the maxillary right molar region distal to the first molar prior to the commencement of orthodontic treatment.

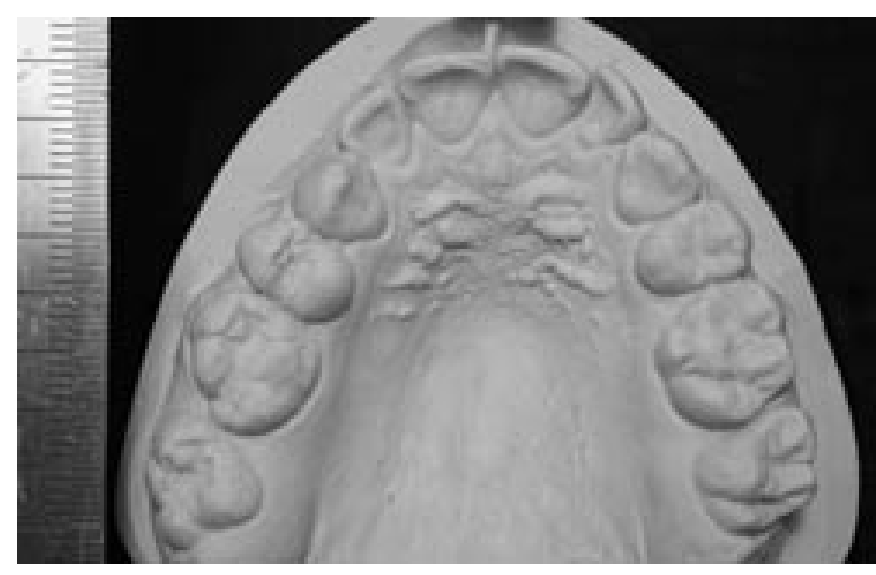

Fig. 1. Occlusal view of the maxillary dentition of the woman.

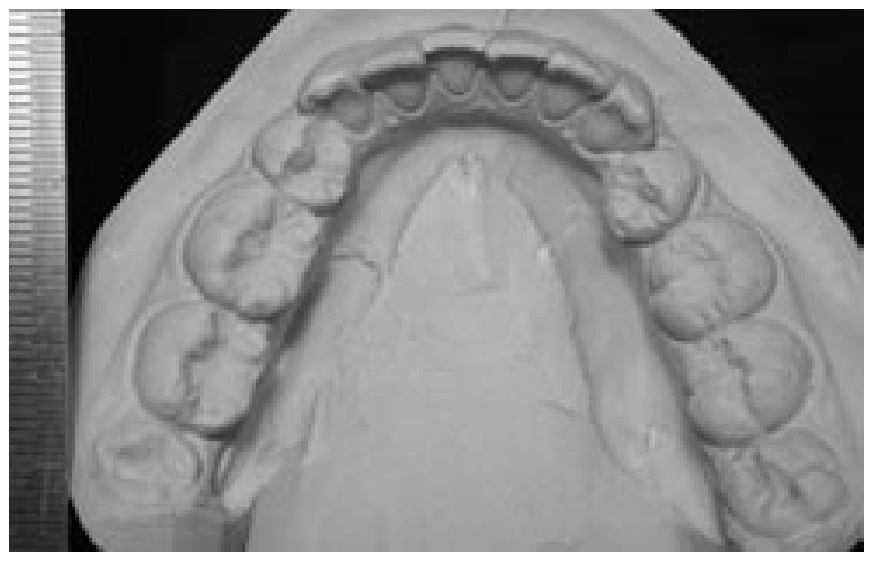

Fig. 2. Occlusal view of the mandibular dentition of the woman.

The maxillary right third molar was not present and the woman confirmed that it had not been extracted. The mandibular left third molar was partly erupted. The maxillary left first and second molars and mandibular left first molar had fissure sealants placed on their surfaces in 2001 and the occlusal surface of the maxillary right first molar had been restored in amalgam in 1994, then the amalgam had been replaced with composite resin in 2001.

The crowns of the maxillary right second premolar and the first and second molars were markedly different in form to those on the left. There was also some minor variation in crown form of the maxillary right canine. The mandibular right second premolar and first, second and third molars all showed different and unusual crown form compared with those on the left. The affected maxillary and mandibular premolar and molar teeth showed similar features, with altered crown shapes and rounded forms with small intercuspal distances. The maxillary right canine crown showed increased labial convexity compared with its antimere, but this variation was less marked than those of the premolars and molars. Intraoral examination did not disclose any hypoplasia or hypocalcification of the enamel of affected teeth.

Examination of a panoramic radiograph obtained at 20 years 5 months of age showed that the maxillary right third molar was congenitally missing (Fig. 3). This film disclosed some differences in the root morphology of the mandibular right first and second molars compared with the corresponding teeth on the left. The roots of the mandibular right first molar appeared to be more slender than those of the mandibular left first molar. The roots of the mandibular right second molar were more curved (like plier handles) than those of its antimere. The buccal roots of the maxillary right molars also appeared to converge more than the corresponding molar roots on the left that displayed a distal curve. The roots of all teeth were fully formed, except for the mandibular left third molar that was distally impacted. 


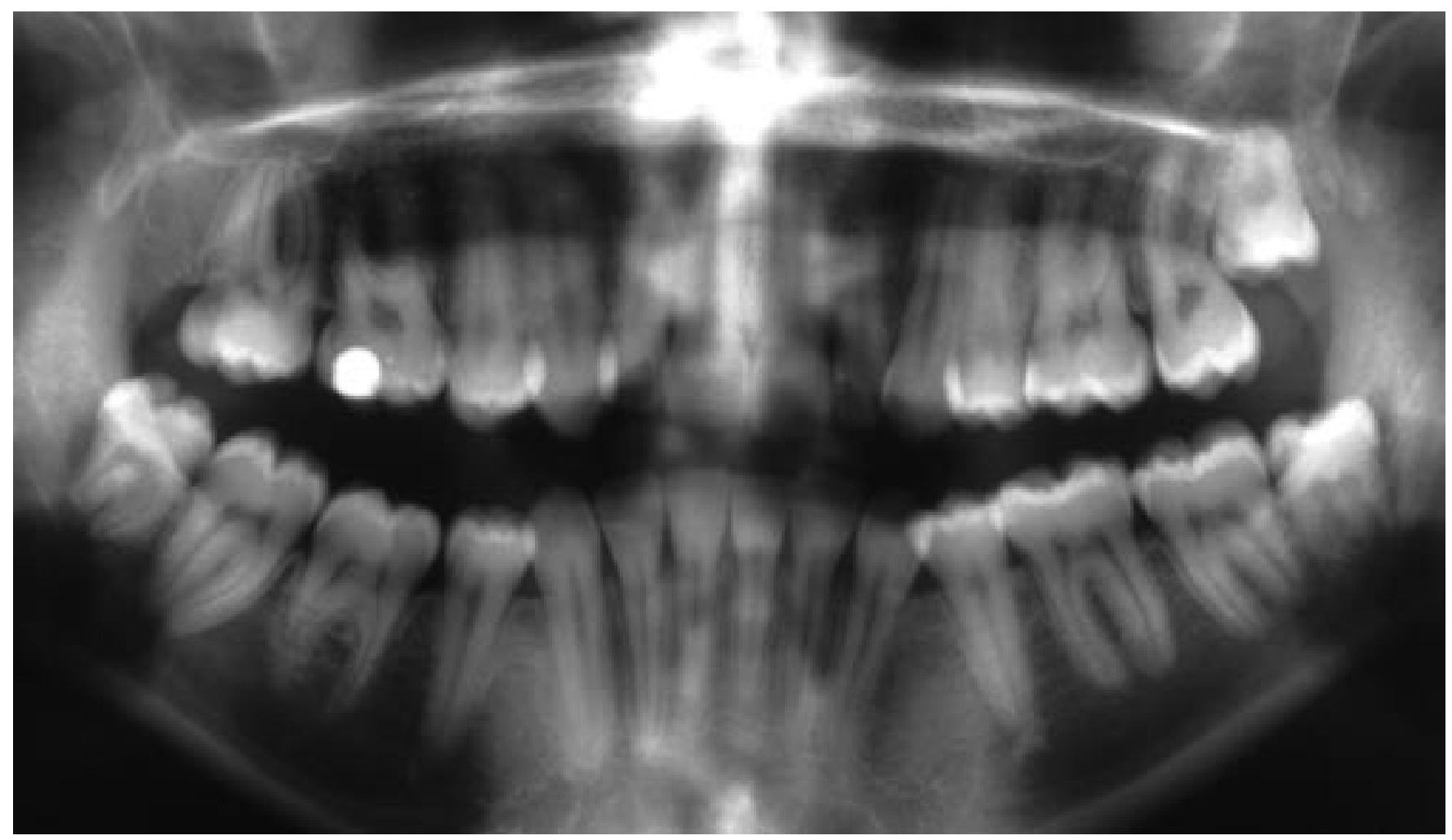

Fig. 3. Panoramic radiograph of the woman.

Bitewing radiographs were also available that enabled an assessment of enamel and dentine thickness and pulp cavity anatomy. The altered contours of the proximal surfaces of affected teeth made it difficult to locate homologous points on the mesial and distal surfaces of antimeric teeth. However, using the methods described by Stroud et al. (1994) and comparing the woman's data with the standards provided by Stroud and colleagues, enamel and dentine thickness fell within the normal ranges and there were only minor differences between the sides.

The sizes of the dental crowns were compared between sides and with normal data published for individuals of European ancestry (Townsend et al., 1986). Maximum mesiodistal and buccolingual crown diameters were recorded according to the definitions of Seipel (1946) and expressed as Z-scores against standards for girls. All of the z-scores except two were positive, indicating that the woman's dental crown size was generally larger than normal. In particular, the z-score for the buccolingual crown diameter of the maxillary right second premolar was 3.5 (compared with 2.8 on the left) and the z-score for the buccolingual crown diameter of the mandibular right first molar was 3.4 (compared with 1.9 on the left). In contrast, the z-score for the mesiodistal crown diameter of the mandibular right first molar was -0.3 (compared with 1.7 on the left) and the z-score for the mesiodistal crown diameter of the mandibular right second molar was 0.1 (compared with 1.0 on the left). Therefore, the mandibular right molars showed markedly reduced mesiodistal crown diameters but increased buccolingual diameters compared with their antimeres.

Intercuspal distances were also recorded for the woman's first molars and maxillary second premolars, then comparisons were made between sides and with unpublished normal values that had been computed previously in our laboratory for a sample of females of European ancestry. The woman's intercuspal distances were expressed as z-scores and all of these values were positive on the left side, consistent with the fact that overall crown size of these teeth was also larger than average. However, the values of $\mathrm{z}$-scores for intercuspal dimensions of the right first molars and the maxillary right second premolar were all negative. They ranged from -0.8 for the distance between the mesiobuccal and distobuccal cusps of the mandibular first molar, to -2.0 for the distance between the mesiobuccal and mesiolingual cusps of the maxillary first molar. These measurements confirmed the visual impression that the cusp tips were closer together on the posterior teeth on the right compared with the left.

The mandibular right first molar was a four-cusped tooth compared with its antimere that displayed the typical five-cusped appearance. There was also altered expression of Carabelli trait between the maxillary right and left first molars, the former displaying a groove form of the feature whereas the latter showed a cuspal 


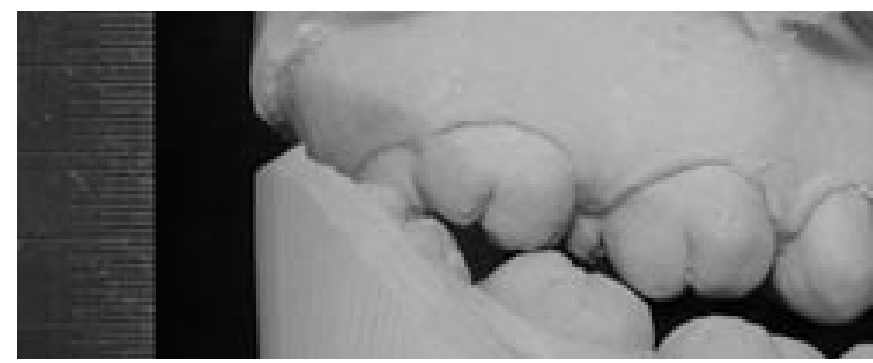

Fig. 4. Right buccal view of the woman's dentition, with models occluded in intercuspal occlusion.

form.

When the dental casts were examined from the buccal view with the teeth in maximum intercuspation, the premolars and first molars on the right did not occlude whereas there was contact between opposing teeth on the left (Figs. 4 and 5). The maxillary right canine was in a crossbite relationship with the mandibular right canine and lateral incisor. The central incisors displayed a normal overbite and overjet relationship (Fig. 6), although the mandibular incisors were retroclined and the mandibular arch midline was displaced $2-3 \mathrm{~mm}$ to the right. Given that orthodontic treatment had been carried out, including extraction of first premolars, we did not attempt to develop a common hypothesis to explain the altered crown form of the premolars and molars, and the posterior open bite, on the right side.

\section{DISCUSSION}

Although some of the woman's teeth showed asymmetry in overall crown size, especially the mandibular first molars, the most striking feature was the asymmetrical expression of crown shape of both maxillary and mandibular posterior teeth. The posterior teeth on the right showed more rounded cuspal outlines with smaller intercuspal distances than their antimeres on the left. The alteration in crown form was localized mainly to the maxillary and mandibular posterior segments, specifically the premolars and molars, although there was some minor variation in the labial convexity of the maxillary right canine. The first premolars had been extracted for orthodontic reasons so it was not possible to examine them. Nor was it possible to examine any of the woman's primary teeth.

Given that there was no indication that the enamel on the affected teeth was hypoplastic or hypocalcified, it would seem that some disturbance must have affected the morphogenesis of the developing premolar and molar tooth germs on the right side only. The location of the cusp tips on premolars and molars is associated with the development of enamel knots in the enamel organ, that is those regions of the internal enamel epithelium that cease mitosis, leading to the buckling of its surface (Thesleff et al., 2001). The final shape of the cusps depends on the subsequent deposition of enamel

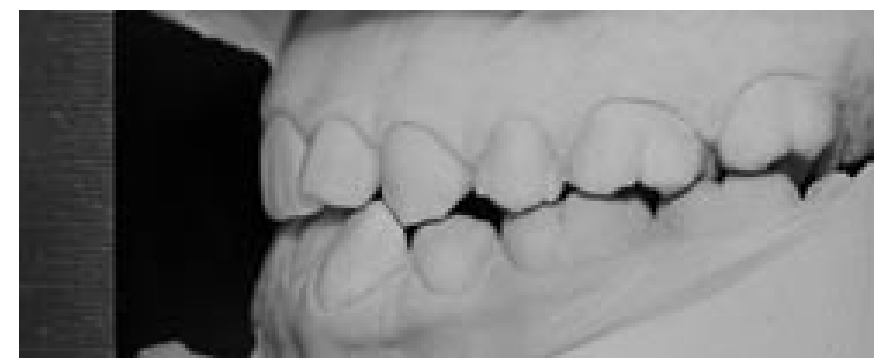

Fig. 5. Left buccal view of the woman's dentition, with models occluded in intercuspal occlusion.

by ameloblasts. As the woman's enamel was apparently normal both qualitatively and quantitatively, the most likely site of the disruption is the internal enamel epithelium.

We have reported that heritability estimates for intercuspal distances of molar teeth derived from a large sample of twins are only moderate in magnitude compared with those for overall crown dimensions (Townsend et al., 2003). Intercuspal distances were also associated with higher coefficients of variation than overall crown measures, confirming that they display relatively greater phenotypic variation than maximum mesiodistal and buccolingual diameters. These results are consistent with the findings of molecular studies (e.g., Tucker and Sharpe, 1999; Thesleff et al., 2001), indicating that epigenetic influences related to the release of specific signalling molecules from the regions of the enamel knots are important in determining how the internal enamel epithelium folds during odontogenesis. It is possible, therefore, that the localized alteration of crown form in this case has resulted from a disruption to the development of enamel knots on one side of the arch. This may have been triggered by traumatic event.

It is difficult to say what the cellular or molecular basis of such a disturbance could be, but it is tempting to suggest that an upset to neural crest cell migration, or to the reciprocal interaction between the ectomesenchymal cells of the dental papilla and the epithelial cells of the internal enamel epithelium, might underlie the problem. It is very unlikely that a genetic mutation has caused the morphological asymmetry, as this would be most likely to affect teeth on both sides of both dentitions. A pos-

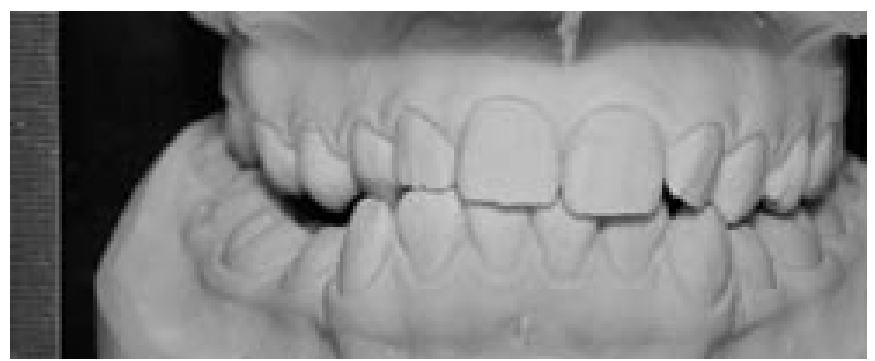

Fig. 6. Labial view of the woman's dentition, with models occluded in intercuspal occlusion. 
sible exception could be mosaicism, with different cell lines regulated by discrete genes producing morphological asymmetry. Although the woman reported here showed no other signs of physical abnormality, we were unable to test for mosaicism.

The observed pattern of morphological variation within the human dentition usually follows Butler's field theory (Dahlberg, 1945; Butler, 2001), with the more distal tooth in each class showing greater variation than more mesially positioned teeth. For example, third molars generally show considerable variation in morphology, much more than first molars. In this case, however, the "key" molar tooth seemed to be affected to the same degree as the more distal members of the class. This tends to confirm that the localized variation in crown form resulted from a distinct, though relatively minor, developmental disturbance and does not merely represent an extreme example of the normal range of development.

Another model that may prove useful in trying to decipher the underlying basis of variation within the human dentition is the so-called "facial homeobox code" described by Sharpe (1995). The homeobox genes in the developing face are restricted to specific domains, with incisor, canine and molar fields being described. As Sharpe (1995) points out, it is possible that neural crest cells are pre-patterned with homeobox genes prior to or during their migration. Subsequent reciprocal interactions between those neural crest cells contributing to the ectomesenchyme of the dental papilla with the epithelial cells of the internal enamel organ would then define tooth type and shape. It is possible that some localized upset to expression of the molar homeobox code has produced the unilateral variation in dental morphology that we have observed in this case.

The timing of onset and duration of crown formation of the affected teeth provide further insights into the possible nature of the disturbance. The crowns of the permanent first molars begin to calcify at around birth, so the period of folding of the internal enamel epithelium is mainly a pre-natal event, although distortion could still occur post-natally until the cusp tips have been united by the spread of calcification. The second premolar crowns commence their calcification at around 2.0 to 2.5 years and the second molars around 2.5 to 3.0 years, so folding and potential distortion of the internal enamel epithelia of these teeth persists into the post-natal period. The third molars may not commence crown calcification until 7-10 years, so there are several years after birth during which disturbances may affect their crown form (Hillson, 1996:123).

Given that all of the affected teeth in this case show similar alterations in their crown form, it would seem that some ongoing localized disturbance in the function of one or more cell lines in the developing teeth is the most likely etiological factor. It is possible that there could have also been superimposed local space constraints that led to the alterations in overall crown shape of the mandibular molars, compressing them mesiodistally but allowing them to grow buccolingually. For example, Taylor (1978:257) has described in detail the appearance of compressed teeth and suggested that their appearance may have resulted from crowding of tooth buds prior to calcification. Space constraints may also account for the apparent differences in molar root form between the sides.

Several researchers have reported on asymmetrical expression of so-called non-metric crown variants, such as Carabelli trait (e.g., Saunders and Mayhall, 1982; Pinkerton et al., 1999). This normal variation may take the form of a large cusp on one side and a smaller cusp on the other, or there may be different expressions of grooves on each side. However, it is rare to find a cuspal form of Carabelli trait on one side but no expression or a small groove on the other. Again, then, the observed expression of Carabelli trait in this case suggests that a specific disturbance has occurred and that the variation in expression does not fall within the so-called normal range of variation.

This case highlights the fact that asymmetries in dental crown form, whether they be fluctuating or directional, need to be viewed as resulting from a continuum of developmental disturbances that may range from minor to severe. As our knowledge of the molecular basis of dental development continues to grow, we should eventually be able to explain in cellular and molecular terms the specific causes of the whole range of asymmetrical expressions in dental crown form that we observe within the human dentition.

\section{LITERATURE CITED}

Butler PM. 2001. What happened to the field theory. In: Brook A, editor. Dental morphology 2001. Sheffield: Sheffield Academic Press, p 3-12.

Dahlberg AA. 1945. The changing dentition of man. Am J Dent Assoc 32:676-690.

Harris EF. 1992. Laterality in human odontometrics: analysis of a contemporary American White series. In: Lukacs JR, editor. Culture, ecology and dental anthropology. Delhi, Kamla-Raj, p 157-170.

Hillson S. 1996. Dental Anthropology. Cambridge: Cambridge University Press, p 123.

Moorrees CFA, Thomsen SO, Jensen E, Yen PKJ. 1957. Mesiodistal crown diameters of human permanent teeth in individuals. J Dent Res 36:39-47.

Narayanan A, Smith S, Townsend G. 1999. Dental crown size in individuals with cleft lip and palate. Perspect Hum Biol 4:61-70.

Pinkerton S, Townsend G, Richards L, Schwerdt W, Dempsey P. 1999. Expression of Carabelli trait in both dentitions of Australian twins. Perspec Hum Biol 4:19-28. 
Pirttiniemi P, Alvesalo L, Silven O, Heikkila J, Julku J, Karjalahti P. 1998. Asymmetry in the occlusal morphology of first permanent molars in $45, \mathrm{X} / 46, \mathrm{XX}$ mosaics. Archs Oral Biol 43:25-32.

Saunders SR, Mayhall J. 1982. Developmental patterns of human dental morphological traits. Archs Oral Biol 27:45-49.

Siegel MI, Doyle WJ, Kelley C. 1977. Heat stress, fluctuating asymmetry and prenatal selection in the laboratory rat. Am J Phys Anthropol 46:121-126

Seipel CM. 1946. Variation of tooth position. Sven Tandlak Tidskr 39: Suppl.

Seow WK, Urban S, Vafaie N, Shusterman S. 1998. Morphometric analysis of the primary and permanent dentitions in hemifacial microsomia: a controlled study. J Dent Res 77:27-38.

Sharpe PT. 1995. Homeobox genes and orofacial development. Connect Tiss Res 32:17-25.

Stroud JL, Buschang PH, Goaz PW. 1994. Sexual dimorphism in mesiodistal dentin and enamel thickness. Dentomaxillofac Radiol 23:169-171.

Taylor RMS. 1978. Variation in the morphology of teeth. Springfield, IL: Charles C Thomas, p 257.

Thesleff I, Keranen S, Jernvall J. 2001. Enamel knots as signalling centers linking tooth morphogenesis and odontoblast differentiation. Adv Dent Res 15:14-18.

Townsend GC. 1983. Fluctuating dental asymmetry in Down's syndrome. Aust Dent J 28:39-44.

Townsend GC and Brown T. 1981. The Carabelli trait in Australian Aboriginal dentition. Archs Oral Biol 26: 809-814.

Townsend G, Dempsey P, Richards L. 1999. Asymmetry in the deciduous dentition: fluctuating and directional components. Perspec Hum Biol 4:45-52.

Townsend G, Richards L, Hughes T. 2003. Molar intercuspal dimensions: genetic input to phenotypic variation. J Dent Res 82:350-355.

Townsend GC, Alvesalo L, Jensen B, Kari M. 1986. Patterns of tooth size in human chromosomal aneuploidies. In: Russell DE, Santoro JP, Sigogneau-Russell D, editors. Teeth revisited: Proceedings of the VIIth International Symposium on Dental Morphology, Mem Mus Natn Hist Nat Paris (series C), Paris, France 53:25-45.

Tucker AS, Sharpe PT. 1999. Molecular genetics of tooth morphogenesis and patterning: the right shape in the right place. J Dent Res 78:826-834.

\section{Decoding Your Subscription}

Want to know when your subscription to Dental Anthropology expires? Membership in the Association and, thus, your subscription to Dental Anthropology is on an annual basis coinciding with the calendar year. Have a look at the mailing label on the evelope that this issue arrived in, and you will see the year for which your dues have been paid. The year is located in parentheses to the right of your name. So, if the mailing label says "(2004)" you are paid to the end of this calendar year.

In order to extend your membership, fill-out the relevant portions of the enclosed form (or e-mail the Secretary) - remember to include appropriate payment-and mail it to the Secretary-Treasurer of the Association:

Dr. Heather H. Edgar

Maxwell Museum of Anthropology

MSC01 1050

1 University of New Mexico

Albuquerque, New Mexico 87131-0001 USA

telephone: (505) 277-4415

e-mail: hjhedgar@unm.edu 